PROSTATE CANCER

\title{
Radiosensitized by STAT5 blockade
}

New data published in Clinical Cancer Research show that inhibition of signal transducer and activator of transcription $5 \mathrm{~A}$ and $5 \mathrm{~B}$ (STAT5) in prostate cancer models suppresses DNA repair via homologous recombination $(\mathrm{HR})$ and increases sensitivity to radiotherapy without inducing similar effects in the gastrointestinal mucosa.

Radiotherapy induces DNA double strand breaks, resulting in cell death in tumour cells with diminished DNA repair capacity. In patients with prostate cancer, adverse effects of this treatment in neighbouring tissues are common. To increase the effectiveness of radiotherapy for prostate cancer and limit its toxicity, researchers investigated whether inhibiting DNA repair would sensitize prostate cancer to radiation, which might enable dose reductions.

RAD51 is essential in HR DNA repair. First, the researchers determined that STAT5 is required for RAD51 expression in prostate cancer, including in patient-derived ex vivo models. Activation of STAT5 increased, whereas inhibition of STAT5 on the genetic level or through a small molecule inhibitor decreased RAD51 expression. Comet assays showed that inhibition of STAT5 by expression of dominant negative STAT5 resulted in decreased repair of radiation-induced DNA damage.

Focusing on the interaction between STAT5 and RAD51, the team found that RAD51 foci formation was decreased following radiation in a prostate cancer cell line in which STAT5 expression had been knocked down. In addition, a green fluorescent protein (GFP) reporter assay of HR DNA repair showed that STAT5 activation increased, whereas inhibition reduced HR DNA repair. In cells that constitutively expressed STAT5, HR DNA repair was increased, which could be reversed by RAD51 knockdown. Notably, STAT5 inhibition did not influence the non-homologous end joining DNA repair pathway.
Investigating sensitization of prostate cancer cells to radiation, the researchers found that both genetic and pharmacological STAT5 inhibition reduced cell survival following radiation in clonogenic assays. Finally, in a prostate cancer xenograft model, combination of radiation with a STAT5 inhibitor suppressed tumour growth more than either treatment alone. By contrast, the combination treatment did not seem to affect morphology of intestinal crypts or induce apoptosis.

Clemens Thoma

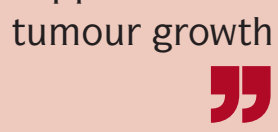

ORIGINAL ARTICLE Maranto, C. et al. STAT5A/B blockade sensitizes prostate cancer to radiation through inhibition of RAD51 and DNA repair. Clin. Cancer Res. https://doi.org/10.1158/1078-0432. CCR-17-2768 (2018)

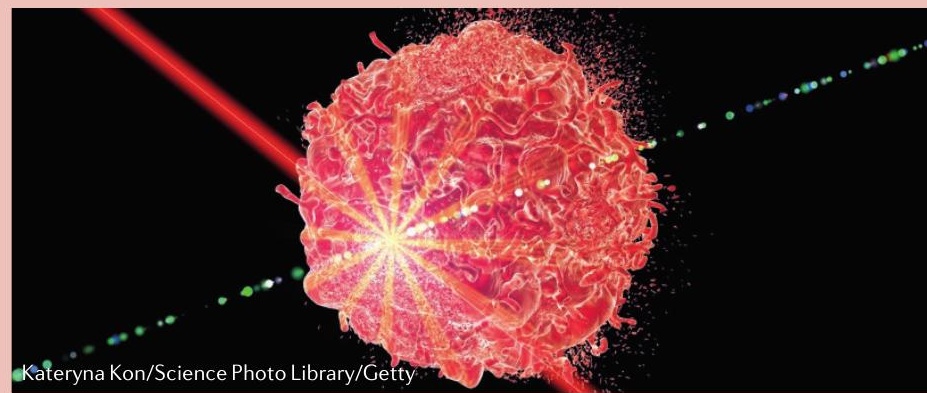

